\title{
Multipartite Einstein-Podolsky-Rosen steering sharing with separable states
}

\author{
Yu Xiang, ${ }^{1,2}$ Xiaolong Su, ${ }^{2,3}$ Ladislav Mišta, Jr., ${ }^{4}$ Gerardo Adesso, ${ }^{5}$ and Qiongyi He ${ }^{1,2,6}$ \\ ${ }^{1}$ State Key Laboratory for Mesoscopic Physics and Collaborative Innovation Center of Quantum Matter, \\ School of Physics, Peking University, Beijing 100871, China \\ ${ }^{2}$ Collaborative Innovation Center of Extreme Optics, Shanxi University, Taiyuan, Shanxi 030006, China \\ ${ }^{3}$ State Key Laboratory of Quantum Optics and Quantum Optics Devices, Institute of Opto-Electronics, \\ Shanxi University, Taiyuan 030006, China \\ ${ }^{4}$ Department of Optics, Palacký University, 17. listopadu 12, 77146 Olomouc, Czech Republic \\ ${ }^{5}$ Centre for the Mathematics and Theoretical Physics of Quantum Non-Equilibrium Systems (CQNE), \\ School of Mathematical Sciences, The University of Nottingham, Nottingham NG7 2RD, United Kingdom \\ ${ }^{6}$ Beijing Academy of Quantum Information Sciences, West Bld. \#3, No. 10 Xibeiwang East Rd., \\ Haidian District, Beijing 100193, China
}

(Received 25 January 2018; published 22 January 2019)

\begin{abstract}
The distribution of quantum correlations among remote users is a key procedure underlying many quantum information technologies. Einstein-Podolsky-Rosen steering, which is one kind of such a correlation stronger than entanglement, has been identified as a resource for secure quantum networks. We show that this resource can be established between two and even more distant parties by transmission of a system being separable from all the parties. For two-user scenarios, we design a protocol allowing one to distribute one-way Gaussian steering which can be used subsequently for one-sided device-independent (1sDI) quantum key distribution. Further, we extend the protocol to three-user scenarios to distribute richer steerability properties including one-to-multimode steering and collective steering which can be used for 1sDI quantum secret sharing. All the proposed protocols can be implemented with squeezed states, beam splitters, and displacements, and thus they can be readily realized experimentally. Our findings reveal that not only entanglement but even steering can be distributed via communication of a separable system. Viewed from a different perspective, the present protocols also demonstrate that one can switch multipartite states between different steerability classes by operations on parts of the states.
\end{abstract}

DOI: 10.1103/PhysRevA.99.010104

Einstein-Podolsky-Rosen (EPR) steering was put forward by Schrödinger [1] to describe the "spooky action-at-adistance" debated in the original EPR paradox [2,3], which allows one observer to adjust ("steer") the state of another remote observer by local measurements. This special type of quantum correlation offers insights into directional nonlocality [4-14] and differs conceptually from inseparable correlations, also known as entanglement $[15,16]$. The fact that steering enables verification of shared entanglement even when one party's measurements are untrusted [17-19] makes it an essential resource for a number of applications, such as one-sided device-independent (1sDI) quantum key distribution (QKD) [20-23] and quantum secret sharing (QSS) [24-26], secure quantum teleportation [27-29], and subchannel discrimination $[30,31]$.

However, in general it is harder to establish EPR steering than entanglement, as the former requires a stronger interaction and tolerates less noise than the latter [6,32]. In a practical quantum network, not all users might have the ability to produce steering, and distributing it directly might expose the transmitted quantum states to the unwanted attacks of an eavesdropper. A more efficient scenario would be to have a quantum cloud server which can generate quantum states and perform appropriate operations for different tasks, and then establish desired correlations between the users, mediated by transmission of ancillary systems with as little as possible quantum resources. Somehow counterintuitively, it has been shown theoretically [33-35] and experimentally [36-38] that entanglement (inseparability) can be distributed between two parties via a separable ancilla. However, the resources needed to distribute more stringent forms of correlations such as EPR steering and Bell nonlocality remained unexplored.

In this Rapid Communication, we show that EPR steering, which is strictly stronger than entanglement and possesses intrinsical asymmetry, can be faithfully distributed between two and even more distant parties with minimal resources in continuous-variable (CV) Gaussian systems. By preparing locally initial quantum states, performing suitably tailored local correlated displacements on them, and transmitting a separable ancilla mode across multiuser networks (see Fig. 1), we show how to establish a plethora of useful steering properties, such as one-way Gaussian steering in two-user scenarios, two-way steering and collective steering which can be used for CV QSS $[39,40]$ in three-user scenarios, and so on. Furthermore, we prove that the distributed steerability among distant users can be maximized by optimal displacements. We further present a modified scheme with a relaxed condition that the ancilla mode used for distribution is nonsteerable instead of separable from the users, yielding a broader range of parameters for which the protocol succeeds. These results 


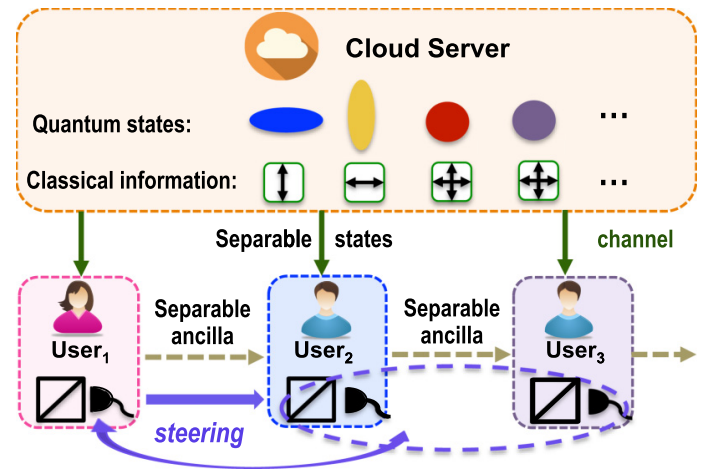

FIG. 1. Scheme to distribute Gaussian EPR steering among different users via a separable ancilla: The quantum cloud server locally produces the quantum states and analyzes the classical information of the displacements required by the task, then sends the separable quantum states to the users. By transmitting a separable ancilla, the users can successfully share EPR steering with desired properties.

shed light on the rich steering sharing structure in multipartite $\mathrm{CV}$ systems and yield experimentally feasible recipes for the scalable realization of $1 \mathrm{sDI}$ communication tasks across multiuser quantum networks.

Gaussian steerability. For any $\left(n_{A}+m_{B}\right)$-mode Gaussian state, we put the amplitude (position) and phase (momentum) quadratures of each mode into a column vector $\hat{\xi}:=\left(\hat{x}_{1}^{A}, \hat{p}_{1}^{A}, \ldots, \hat{x}_{n_{A}}^{A}, \hat{p}_{n_{A}}^{A}, \hat{x}_{1}^{B}, \hat{p}_{1}^{B}, \ldots, \hat{x}_{m_{B}}^{B}, \hat{p}_{m_{B}}^{B}\right)^{\top}$, satisfying the canonical commutation rules $\left[\hat{x}_{j}, \hat{p}_{k}\right]=2 i \delta_{j k}$. The properties of the state can be fully specified by its covariance matrix (CM) $\gamma_{A B}$ with elements $\left(\gamma_{A B}\right)_{i j}=$ $\left\langle\hat{\xi}_{i} \hat{\xi}_{j}+\hat{\xi}_{j} \hat{\xi}_{i}\right\rangle / 2-\left\langle\hat{\xi}_{i}\right\rangle\left\langle\hat{\xi}_{j}\right\rangle$, which reads as $\gamma_{A B}=\left(\begin{array}{cc}A & C \\ C^{\top} & B\end{array}\right)$. Here, the submatrices $A$ and $B$ are the CMs corresponding to the reduced states of each subsystem, respectively. The steerability from Alice to Bob via Gaussian measurements can be quantified by [7]

$$
\mathcal{G}^{A \rightarrow B}\left(\gamma_{A B}\right):=\max \left\{0,-\sum_{j: \bar{v}_{j}^{A B \backslash A}<1} \ln \left(\bar{v}_{j}^{A B \backslash A}\right)\right\},
$$

where $\bar{v}_{j}^{A B \backslash A}\left(j=1, \ldots, m_{B}\right)$ are the symplectic eigenvalues of the Schur complement of $A$ defined as $\bar{\gamma}_{A B \backslash A}=B-$ $C^{\top} A^{-1} C$. The quantity $\mathcal{G}^{A \rightarrow B}$ is a monotone under Gaussian local operations and classical communication [41] and vanishes when Alice cannot steer Bob by Gaussian measurements $[7,42]$. This quantifier has been experimentally measured in Gaussian cluster states by reconstructing the CM [13].

For the sake of experimental feasibility, one can also confirm the presence of steering when the EPR variance product $E_{B \mid A}:=\Delta_{\inf , A} \hat{x}_{B} \Delta_{\inf , A} \hat{p}_{B}<1$. Here, $\Delta_{\text {inf, }{ }_{A}} \hat{x}_{B}=$ $\Delta\left(\hat{x}_{B}-g_{x} \hat{x}_{A}\right)$ is the minimum inferred variance of Bob's position outcome given Alice's result with optimal gain factor $g_{x}=\left\langle\hat{x}_{B}, \hat{x}_{A}\right\rangle /\left(\Delta \hat{x}_{A}\right)^{2}[6]$, where $\langle x, y\rangle:=\langle x y\rangle-\langle x\rangle\langle y\rangle$, and $\Delta_{\text {inf, } A} \hat{p}_{B}$ is defined similarly. The criterion $E_{B \mid A}<1$ is necessary and sufficient to test steering by Gaussian measurements. The quantity $E_{B \mid A}$, which can be efficiently measured by homodyne detection [24], in fact directly quantifies the Gaussian steerability, as $\mathcal{G}^{A \rightarrow B}=\max \left\{0,-\ln E_{B \mid A}\right\}$ for all

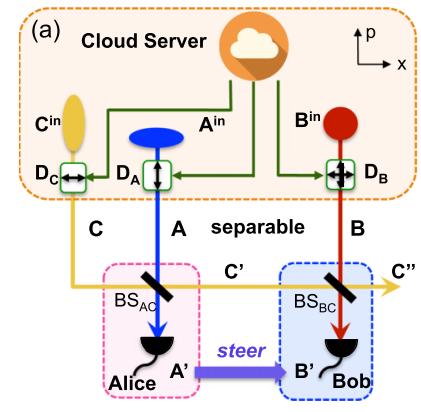

(b)

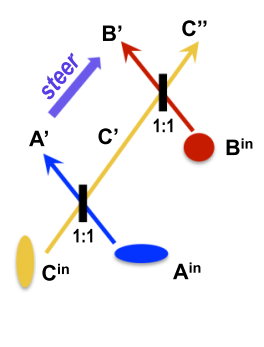

FIG. 2. (a) Sketch of the one-way Gaussian steering distribution protocol. See text for details. The best steerability is recovering the steerability in the tripartite entangled state created by the optical network, as illustrated in (b).

two-mode CMs $\gamma_{A B}$ in standard form (i.e., with diagonal $A, B, C)[7,26]$.

In the following, we first show that one-way steering can be distributed from Alice to Bob by a separable ancilla. We improve the protocol developed for entanglement [35] by optimizing the displacements to distribute the highest steerability. As depicted in Fig. 2(a), the initial modes $A, B$, and $C$ sent from the cloud server are in a fully separable state, and the ancillary mode $C^{\prime}$ is separable from the modes held by Alice and Bob, ensuring the security of the process of establishing Gaussian steering. We prove that the highest steerability which can be distributed by a separable ancilla is determined by the utilized optical network composed only of input squeezed states and beam splitters, as depicted in Fig. 2(b).

Protocol. The protocol consists of three steps. In step 1 , the cloud server initially produces modes $A^{\text {in }}$ and $C^{\text {in }}$ in momentum- and position-squeezed vacuum states, respectively, while mode $B^{\text {in }}$ is in a vacuum state. All three modes are in a product state described by the CM $\gamma_{A B C}^{\text {in }}=\operatorname{diag}\left(e^{2 t}, e^{-2 t}, 1,1, e^{-2 t}, e^{2 t}\right)$, where $t$ is the squeezing parameter. The three modes are then appropriately displaced by local correlated displacements $\hat{p}_{A^{\text {in }}} \rightarrow \hat{p}_{A^{\text {in }}}-$ $D_{A} p_{d}, \quad \hat{x}_{C^{\text {in }}} \rightarrow \hat{x}_{C^{\text {in }}}+D_{C} x_{d}, \quad \hat{x}_{B^{\text {in }}} \rightarrow \hat{x}_{B^{\text {in }}}+D_{B} x_{d}, \quad \hat{p}_{B^{\text {in }}} \rightarrow$ $\hat{p}_{B^{\text {in }}}+D_{B} p_{d}$. Here, $x_{d}$ and $p_{d}$ obey a zero mean Gaussian distribution with the same variance and $D_{A}, D_{B}, D_{C}$ are the strengths of the displacements, to be specified in the second step. The resulting state is fully separable with $\mathrm{CM} \gamma_{A B C}=$ $\gamma_{A B C}^{\text {in }}+\tilde{D}$, where $\tilde{D}$ denotes a positive noise matrix created by the displacements.

In step 2, Alice superimposes modes $A$ and $C$ on a balanced beam splitter $B S_{A C}$, thereby creating a three-mode state with CM $\gamma_{A^{\prime} B C^{\prime}}=U_{A C}\left(\gamma_{A B C}^{\text {in }}+\tilde{D}\right) U_{A C}^{\top}=U_{A C} \gamma_{A B C}^{\text {in }} U_{A C}^{\top}+$ $x P, x \geqslant 0$. Here, the symplectic matrix $U_{A C}$ describes the beam splitter, and $U_{A C} \gamma_{A B C}^{\text {in }} U_{A C}^{\top}$ describes a product state of vacuum mode $B^{\text {in }}$ and a two-mode squeezed state obtained by mixing the undisplaced squeezed modes $A^{\text {in }}$ and $C^{\text {in }}$ at the beam splitter [see Fig. 2(b)]. However, the entanglement between the output modes $A^{\prime}$ and $C^{\prime}$ can be smeared by adding a sufficiently large noise term $x P$, where $P$ is a suitable positive matrix and $x$ regulates the strength of noise. Using the method developed in Ref. [44], the matrix $P$ can be constructed as $P=q_{1} q_{1}^{\top}+q_{2} q_{2}^{\top}$ from the $6 \times 1$ vectors 
$q_{1}=\left(0,-1,0, d_{B}, 0,-1\right)^{\top}$ and $q_{2}=\left(1,0, d_{B}, 0,-1,0\right)^{\top}$ to negate the entanglement between $A^{\prime}$ and $C^{\prime}$, where the parameter $d_{B}$ can be optimized to reach the highest steerability from Alice to Bob in the final state. The CM of the state after step 2 becomes

$$
\gamma_{A^{\prime} B C^{\prime}}=\left(\begin{array}{ccc}
m \mathbb{1} & d_{B} x \sigma_{z} & n \sigma_{z} \\
d_{B} x \sigma_{z} & \left(1+d_{B}^{2} x\right) \mathbb{1} & -d_{B} x \mathbb{1} \\
n \sigma_{z} & -d_{B} x \mathbb{1} & m \mathbb{1}
\end{array}\right),
$$

where $m=\cosh 2 t+x$ and $n=\sinh 2 t-x$. Hence, one can determine the correlation matrix of displacements $\tilde{D}$ prior to the beam splitter $B S_{A C}$ as $\tilde{D}=x U_{A C}^{\top} P U_{A C}$. This corresponds to displacement strengths $D_{A}=D_{C}=\sqrt{2}, D_{B}=d_{B}$, and displacement variances $\left\langle\left(\Delta x_{d}\right)^{2}\right\rangle=\left\langle\left(\Delta p_{d}\right)^{2}\right\rangle=x$. The free parameters $d_{B}$ and $x$ need to be suitably adjusted for the protocol to work, as done in the next step.

In step 3, Bob mixes mode $C^{\prime}$ sent by Alice with his mode $B$ on the balanced beam splitter $B S_{B C}$, which yields the final $\mathrm{CM} \gamma_{A^{\prime} B^{\prime} C^{\prime \prime}}=U_{B C} \gamma_{A^{\prime} B C^{\prime}} U_{B C}^{\top}$ in the form

$$
\gamma_{A^{\prime} B^{\prime} C^{\prime \prime}}=\left(\begin{array}{ccc}
m \mathbb{1} & \frac{d_{B} x+n}{\sqrt{2}} \sigma_{z} & \frac{d_{B} x-n}{\sqrt{2}} \sigma_{z} \\
\frac{d_{B} x+n}{\sqrt{2}} \sigma_{z} & \frac{1+m+d_{B} x\left(d_{B}-2\right)}{2} \mathbb{1} & \frac{1+d_{B}^{2} x-m}{2} \mathbb{1} \\
\frac{d_{B} x-n}{\sqrt{2}} \sigma_{z} & \frac{1+d_{B}^{2} x-m}{2} \mathbb{1} & \frac{1+m+d_{B} x\left(d_{B}+2\right)}{2} \mathbb{1}
\end{array}\right) .
$$

Now, by quantifying the amount of the distributed Gaussian steering from mode $A^{\prime}$ to mode $B^{\prime}$ via Eq. (1), we get

$$
\mathcal{G}^{A^{\prime} \rightarrow B^{\prime}}=\ln [2 \cosh 2 t /(\cosh 2 t+1)],
$$

with optimal displacement $d_{B}^{\text {opt }}=\tanh 2 t+1$. Note that $\mathcal{G}^{A^{\prime} \rightarrow B^{\prime}}>0$ for any $t>0$. Interestingly, the right-hand side of Eq. (4) equals the amount of steerability from $A^{\prime}$ to $B^{\prime}$ in the scheme without any displacements, shown in Fig. 2(b) [45]. This means that the optimal displacements ensure separability of the transmitted ancilla from the other modes, while not reducing the maximum steering that can be distributed. Experimentally, one can verify the Gaussian steerablity via the minimum EPR variance product $E_{B^{\prime} \mid A^{\prime}}=$ $(\cosh 2 t+1) /(2 \cosh 2 t)$ by homodyne detection. One finds $E_{B^{\prime} \mid A^{\prime}}<1$ for any $t>0$.

Discussion. From Eq. (4), we find that the Gaussian steerability from Alice to Bob with displacement $d_{B}^{\text {opt }}$ can be distributed for any $t>0$; however, we need also check the separability of the states in steps 2 and 3 to assure that the transmitted ancilla stays separable from the rest at all stages.

After step 2, the shared state transforms from a fully separable state to a two-mode biseparable state following the classification of Ref. [44]. Making use of the positive partial transpose (PPT) criterion [46] one finds that the state with $\mathrm{CM}$ (2) is entangled across $A^{\prime}-$ $\left(B C^{\prime}\right)$ splitting for any $x>0$ and $t>0$, but it is separable with respect to $C^{\prime}-\left(A^{\prime} B\right)$ splitting when $x \geqslant$ $x_{C^{\prime}-\left(A^{\prime} B\right)}=2 \cosh ^{2} 2 t \sinh t /(\cosh t+\cosh 3 t+\sinh t)$ [see the blue solid curve in Fig. 3(a)], and furthermore, it is separable with respect to $B-\left(A^{\prime} C^{\prime}\right)$ splitting for any $x>0$ and $t>0$. Since steering is strictly stronger than entanglement, the CM (2) also represents a state that is nonsteerable
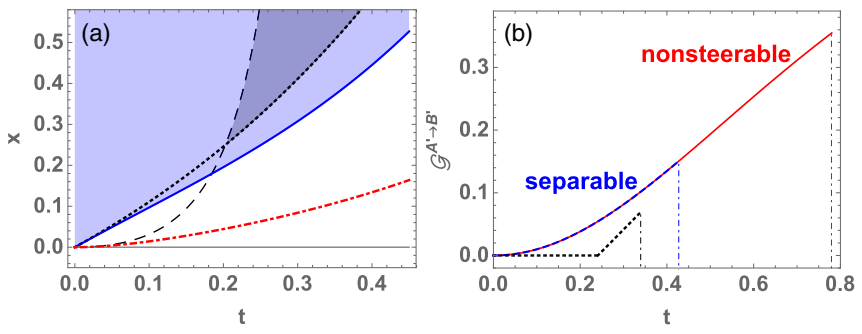

FIG. 3. (a) The blue solid curve represents the threshold of $x_{C^{\prime}-\left(A^{\prime} B\right)}$, above which the state is separable with respect to $C^{\prime}-$ $\left(A^{\prime} B\right)$ splitting. Since $\mathcal{G}^{A^{\prime} \rightarrow B^{\prime}}>0$ for any $t>0$ with optimal $d_{B}^{\text {opt }}$, the parameters above this curve can be used to distribute Gaussian steering by transmitting a separable mode $C^{\prime}$. With a nonoptimal $d_{B}=2$, a much narrower range of parameters within the dark blue (dark gray) area between $x_{A^{\prime} \rightarrow B^{\prime}}$ (black dashed) and $x_{\text {sep }}$ (black dotted) can be used to distribute steering from $A^{\prime}$ to $B^{\prime}$. The red dasheddotted curve shows a relaxed threshold for absence of steering instead of entanglement across $C^{\prime}-\left(A^{\prime} B\right)$ splitting in step 2, above which one can use a nonsteerable mode $C^{\prime}$ to distribute steering. (b) The distributed steerability $\mathcal{G}^{A^{\prime} \rightarrow B^{\prime}}$ for $x=0.5$ in the protocol with optimal $d_{B}^{\text {opt }}$ by a separable (blue dashed) or nonsteerable (red solid) ancilla, and in the case of nonoptimal $d_{B}=2$ (black dotted).

with respect to $C^{\prime}-\left(A^{\prime} B\right)$ splitting if $x \geqslant x_{C^{\prime}-\left(A^{\prime} B\right)}$ and $B-\left(A^{\prime} C^{\prime}\right)$ splitting for any $x>0$ and $t>0$. However, the steerability $\mathcal{G}^{A^{\prime} \rightarrow\left(B C^{\prime}\right)}>0$ for all $x>0$ and $t>0$, which is essential for the performance of the steering distribution from Alice to Bob in the final state. Without the help of the transmitted mode $C^{\prime}$, the second beam splitter alone cannot create steering. In the blue (light gray) area above the blue solid curve $x \geqslant x_{C^{\prime}-\left(A^{\prime} B\right)}$, the state after step 3 described by the CM (3) remains separable with respect to $C^{\prime \prime}-\left(A^{\prime} B^{\prime}\right)$ splitting. Therefore, in this area, the ancilla mode is separable from the rest at all stages, nevertheless, for the Gaussian steerablity of the final state one gets $\mathcal{G}^{A^{\prime} \rightarrow B^{\prime}}>0$ for any $t>0$, which means that Gaussian steering is successfully distributed from Alice to Bob. If we relax the condition that the ancilla is separable to that it is nonsteerable from the rest (i.e., it may be entangled), then the distribution task can be accomplished in an even larger region of parameters, as shown in the area above the red dashed-dotted curve in Fig. 3(a).

Figure 3(b) shows the amount of one-way Gaussian steerability that can be distributed via sending a separable ancilla (blue dashed) and a nonsteerable ancilla (red solid), respectively. One can find that both of them distribute equal steerability from Alice to Bob, but work at two different ranges of initial squeezing parameter $t$ for a fixed value $x=0.5$. By sending a nonsteerable ancilla, the initial squeezing level is requested to satisfy $0<t<0.78$ to guarantee that the transmitted mode $C^{\prime}$ is nonsteerable from $\left(A^{\prime} B\right)$ at all stages, while by sending a separable ancilla, the initial squeezing level is requested to satisfy a more stringent inequality $0<t<0.43$.

Comparing previous results with a nonoptimal displacement, say, $d_{B}=2$ [35], one finds that the distribution of steering via a separable ancilla can only work in the range of $x_{\text {sep }}=\left(e^{2 t}-1\right) / 2 \leqslant x<x_{A^{\prime} \rightarrow B^{\prime}}=\left(1-e^{2 t}\right)^{2} /\left(4-2 e^{2 t}\right)$, depicted by the dark blue (dark gray) area in Fig. 3(a), which 


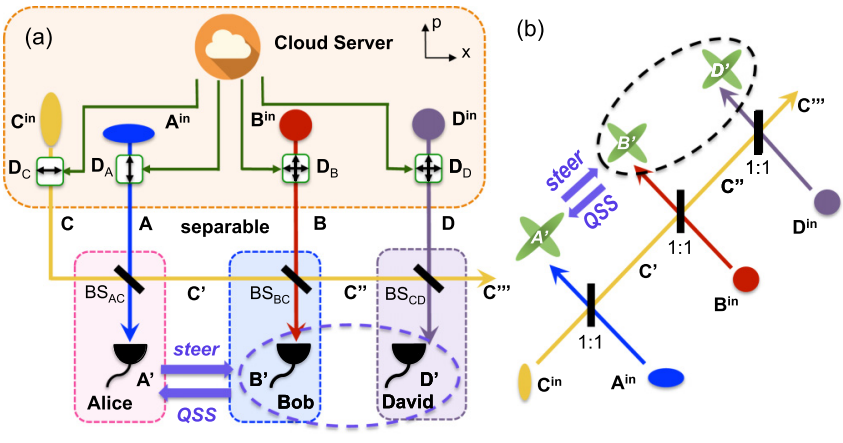

FIG. 4. (a) Scheme for distribution of tripartite Gaussian steering via a separable state. (b) Optical network with the same amount of steering as the optimal scheme with displacements.

is much narrower than the area corresponding to the optimal $d_{B}^{\text {opt }}$. In addition, the distributed steerability [black dotted in Fig. 3(b)] is also lower than that given by the optimal protocol (blue dashed curve). For a fixed value of $x=0.5$, the protocol with $d_{B}=2$ can only work for squeezings obeying $0.241<$ $t<0.346$, thus requiring a nontrivial threshold as opposed to the condition $t>0$ for optimal $d_{B}^{\text {opt }}$.

We have discussed the distribution of Gaussian steering from Alice to Bob with a separable or nonsteerable ancilla. Can the distributed state simultaneously display also Gaussian steering from Bob to Alice in the setup given above? The answer is no. According to the CM (3), mode $B^{\prime}$ and mode $C^{\prime \prime}$ are completely symmetric in the final state. Due to the monogamy relation of Gaussian steering with two observables $\hat{x}$ and $\hat{p}$ [47], neither of them can steer mode $A^{\prime}$, so that only one-way Gaussian steering from Alice to Bob is distributed using the above setup. If Bob wants to steer Alice, he can send the request to the cloud server, and the server can simply switch the initial quantum states and displacements for Alice and Bob.

Multiuser distribution. The scheme can be extended to the multiuser case as shown in Fig. 4(a). Bob continues to send the separable mode $C^{\prime \prime}$ to David who mixes it with his mode $D$ on a balanced beam splitter $B S_{C D}$. It not only distributes tripartite steering from Alice to Bob and David, but also creates a collective steering in the opposite direction.

To accomplish steering distribution in the direction $A^{\prime} \rightarrow$ $B^{\prime} D^{\prime}$, apart from the condition $x \geqslant x_{C^{\prime}-\left(A^{\prime} B\right)}$ that assures the mode $C^{\prime}$ to be separable from modes $\left(A^{\prime} B\right)$ after step 2 , we also need to find further constraints on $x$ and $t$ guaranteeing that the ancilla mode $C^{\prime \prime}$ is separable from subsystem $\left(A^{\prime} B^{\prime} D\right)$ when $x \geqslant x_{C^{\prime \prime}-\left(A^{\prime} B^{\prime} D\right)}$ after step 3 . Besides, we also need to suitably adjust $d_{D}$ in the displacement vectors $q_{1}=\left(0,-1,0, d_{B}^{\text {opt }}, 0,-1,0, d_{D}\right)^{\top}$ and $q_{2}=$ $\left(1,0, d_{B}^{\text {opt }}, 0,-1,0, d_{D}, 0\right)^{\top}$ to distribute steerability as large as possible. The $\mathrm{CM}$ of the resulting four-mode state is detailed in the Supplemental Material [45]. One can prove that with optimal displacement $d_{D}^{\text {opt }}=\sqrt{2} d_{B}^{\text {opt }}$, the highest distributed steerability reads $\mathcal{G}^{A^{\prime} \rightarrow B^{\prime} D^{\prime}}=\ln [4 \cosh 2 t /(3+$ $\cosh 2 t)], \mathcal{G}^{A^{\prime} \rightarrow D^{\prime}}=\ln [4 \cosh 2 t /(1+3 \cosh 2 t)], \mathcal{G}^{A^{\prime} \rightarrow B^{\prime}}=$ $\ln [2 \cosh 2 t /(1+\cosh 2 t)]$, and it can be achieved for any $t>0$. Since $x_{C^{\prime}-\left(A^{\prime} B\right)}>x_{C^{\prime \prime}-\left(A^{\prime} B^{\prime} D\right)}$ reported in Ref. [45], in the blue area in Fig. 3(a) one can perfectly restore the steering of $A^{\prime} \rightarrow B^{\prime} D^{\prime}, A^{\prime} \rightarrow B^{\prime}$, and $A^{\prime} \rightarrow D^{\prime}$ generated by the displacement-free optical network shown in Fig. 4(b) [45].

For the opposite direction $B^{\prime} D^{\prime} \rightarrow A^{\prime}$, keeping $d_{B}^{\text {opt }}$ we can distribute the maximum steerability $\mathcal{G}^{B^{\prime} D^{\prime} \rightarrow A^{\prime}}=$ $\ln [(1+3 \cosh 2 t) /(3+\cosh 2 t)]$ with $d_{D}^{\text {opt }}=(2+2 \operatorname{coth} t+$ $\tanh t-\tanh 2 t) / \sqrt{2}$, which is recovering the steering created in Fig. 4(b). Note that the protocol works only when $t \geqslant 0.943$ and $x \geqslant \max \left\{x_{C^{\prime}-\left(A^{\prime} B\right)}, x_{C^{\prime \prime}-\left(A^{\prime} B^{\prime} D\right)}\right\}=x_{C^{\prime \prime}-\left(A^{\prime} B^{\prime} D\right)}$ [45] to assure the state to be separable with respect to $C^{\prime}-\left(A^{\prime} B\right)$ splitting, as well as $C^{\prime \prime}-\left(A^{\prime} B^{\prime} D\right)$ splitting. For smaller $t$, we need to optimize $d_{B}$ and $d_{D}$ simultaneously. We find that when $0.28 \leqslant t<0.943$, the distributed steering $\mathcal{G}^{B^{\prime} D^{\prime} \rightarrow A^{\prime}}$ can be still maximized by choosing some numerically optimized displacements $d_{B}$ and $d_{D}$, while when $t<0.28$, it is impossible to achieve the same amount of steerability as in the scheme in Fig. 4(b) [45]. In this case, $\mathcal{G}^{B^{\prime} \rightarrow A^{\prime}}=\mathcal{G}^{D^{\prime} \rightarrow A^{\prime}}=0$, which means that neither Bob nor David can individually steer Alice, but they can do that only if they collaborate.

This makes the state a perfect resource for 1sDI QSS, where Alice does not trust Bob and David's devices. Assume Alice acts as the dealer who sends a secret encoded in her state, while Bob and David are players aiming at decoding the message together. To provide security against eavesdropping, a guaranteed secret key rate for the QSS protocol is given by $K \geqslant \ln \left[2 /\left(e E_{A^{\prime} \mid B^{\prime} D^{\prime}}\right)\right]=\mathcal{G}^{B^{\prime} D^{\prime} \rightarrow A^{\prime}}-\ln (e / 2)[25,26]$. A state whose correlations fulfill $K>0$ is certified as a useful resource. Referring to the studied scheme, the condition translates into $\cosh 2 t>(3 e-2) /(6-e)$, which means that a squeezing level of $5.4 \mathrm{~dB}(t>0.62)$, is required to ensure a nonzero key rate. This is well within the current experimental feasibility, since up to $10 \mathrm{~dB}$ of squeezing has been demonstrated [48,49].

Conclusions. We proposed a protocol for the distribution of Gaussian EPR steering across multiuser networks with a separable ancilla. Rich steering properties, such as oneway, one-to-multimode, and collective Gaussian steering, can be distributed via local operations on parts of an initially fully separable state and communication of a separable part of the state. In particular, we derive analytical thresholds on the displacements as a function of the squeezing degree of the initial states such that the protocol succeeds, and prove that the largest steerability that can be distributed recovers that of the multimode states created by the same optical network without correlated displacements. The proposed protocols can be implemented by performing suitable local correlated displacements on the input states of linear optical networks usually used to generate multipartite CV entangled states and hence they are feasible with current technology. Realization of the protocols in the laboratory is particularly attractive in view of the fact that previous experiments on Gaussian entanglement distribution by separable states $[37,38,50]$ were unable to distribute any steering. While our study focused on CV systems and Gaussian measurements, future interesting work can be devoted to steering distribution protocols for qubits as well, building on the seminal study of entanglement distribution with separable states [33]. It would also be interesting to examine our result by applying other feasible criteria, such as the steering inequality with tolerance for measurement-setting errors [51], for non-Gaussian systems. 
Acknowledgments. Y.X., X.S., and Q.H. acknowledge support of the National Key R\&D Program of China (Grants No. 2016YFA0301302, No. 2018YFB1107200, and No. 2016YFA0301402) and the National Natural Science Foundation of China (Grants No. 11622428, No. 11522433, No.
61475006, No. 61675007, and No. 61475092). X.S. thanks the program of Youth Sanjin Scholar and the Fund for Shanxi "1331 Project" Key Subjects Construction. G.A. acknowledges funding from the European Research Council (Starting Grant No. 637352 GQCOP).
[1] E. Schrödinger, Discussion of probability relations between separated systems, Proc. Cambridge Philos. Soc. 31, 555 (1935).

[2] A. Einstein, B. Podolsky, and N. Rosen, Can quantummechanical description of physical reality be considered complete?, Phys. Rev. 47, 777 (1935).

[3] M. D. Reid, Demonstration of the Einstein-Podolsky-Rosen paradox using nondegenerate parametric amplification, Phys. Rev. A 40, 913 (1989).

[4] M. D. Reid, P. D. Drummond, W. P. Bowen, E. G. Cavalcanti, P. K. Lam, H. A. Bachor, U. L. Andersen, and G. Leuchs, Colloquium: The Einstein-Podolsky-Rosen paradox: From concepts to applications, Rev. Mod. Phys. 81, 1727 (2009).

[5] S. L. W. Midgley, A. J. Ferris, and M. K. Olsen, Asymmetric Gaussian steering: When Alice and Bob disagree, Phys. Rev. A 81, 022101 (2010); S. P. Walborn, A. Salles, R. M. Gomes, F. Toscano, and P. H. Souto Ribeiro, Revealing Hidden EinsteinPodolsky-Rosen Nonlocality, Phys. Rev. Lett. 106, 130402 (2011); J. Schneeloch, C. J. Broadbent, S. P. Walborn, E. G. Cavalcanti, and J. C. Howell, Einstein-Podolsky-Rosen steering inequalities from entropic uncertainty relations, Phys. Rev. A 87, 062103 (2013); J. Bowles, T. Vertesi, M. T. Quintino, and N. Brunner, One-way Einstein-Podolsky-Rosen steering, Phys. Rev. Lett. 112, 200402 (2014); B. Opanchuk, L. Arnaud, and M. D. Reid, Detecting faked continuous-variable entanglement using one-sided device-independent entanglement witnesses, Phys. Rev. A 89, 062101 (2014).

[6] Q. Y. He, Q. H. Gong, and M. D. Reid, Classifying Directional Gaussian Entanglement, Einstein-Podolsky-Rosen Steering, and Discord, Phys. Rev. Lett. 114, 060402 (2015).

[7] I. Kogias, A. R. Lee, S. Ragy, and G. Adesso, Quantification of Gaussian Quantum Steering, Phys. Rev. Lett. 114, 060403 (2015).

[8] L. Rosales-Zárate, R. Y. Teh, S. Kiesewetter, A. Brolis, K. $\mathrm{Ng}$, and M. D. Reid, Decoherence of Einstein-Podolsky-Rosen steering, J. Opt. Soc. Am. B 32, A82 (2015).

[9] V. Händchen, T. Eberle, S. Steinlechner, A. Samblowski, T. Franz, R. F. Werner, and R. Schnabel, Observation of oneway Einstein-Podolsky-Rosen steering, Nat. Photon. 6, 596 (2012).

[10] S. Wollmann, N. Walk, A. J. Bennet, H. M. Wiseman, and G. J. Pryde, Observation of Genuine One-Way Einstein-PodolskyRosen Steering, Phys. Rev. Lett. 116, 160403 (2016).

[11] K. Sun, X. J. Ye, J. S. Xu, X. Y. Xu, J. S. Tang, Y. C. Wu, J. L. Chen, C. F. Li, and G. C. Guo, Experimental Quantification of Asymmetric Einstein-Podolsky-Rosen Steering, Phys. Rev. Lett. 116, 160404 (2016).

[12] Y. Xiao, X. J. Ye, K. Sun, J. S. Xu, C. F. Li, and G. C. Guo, Demonstration of Multisetting One-Way Einstein-PodolskyRosen Steering in Two-Qubit Systems, Phys. Rev. Lett. 118, 140404 (2017).
[13] X. W. Deng, Y. Xiang, C. Tian, G. Adesso, Q. Y. He, Q. H. Gong, X. L. Su, C. D. Xie, and K. C. Peng, Demonstration of Monogamy Relations for Einstein-Podolsky-Rosen Steering in Gaussian Cluster States, Phys. Rev. Lett. 118, 230501 (2017).

[14] R. Gallego and L. Aolita, Resource Theory of Steering, Phys. Rev. X 5, 041008 (2015).

[15] H. M. Wiseman, S. J. Jones, and A. C. Doherty, Steering, Entanglement, Nonlocality, and the Einstein-Podolsky-Rosen Paradox, Phys. Rev. Lett. 98, 140402 (2007).

[16] E. G. Cavalcanti, S. J. Jones, H. M. Wiseman, and M. D. Reid, Experimental criteria for steering and the Einstein-PodolskyRosen paradox, Phys. Rev. A 80, 032112 (2009).

[17] S. J. Jones, H. M. Wiseman, and A. C. Doherty, Entanglement, Einstein-Podolsky-Rosen correlations, Bell nonlocality, and steering, Phys. Rev. A 76, 052116 (2007).

[18] E. G. Cavalcanti, M. J. W. Hall, and H. M. Wiseman, Entanglement verification and steering when Alice and Bob cannot be trusted, Phys. Rev. A 87, 032306 (2013).

[19] D. Cavalcanti and P. Skrzypczyk, Quantum steering: A review with focus on semidefinite programming, Rep. Prog. Phys. 80, 024001 (2017).

[20] M. Tomamichel and R. Renner, Uncertainty Relation for Smooth Entropies, Phys. Rev. Lett. 106, 110506 (2011).

[21] C. Branciard, E. G. Cavalcanti, S. P. Walborn, V. Scarani, and H. M. Wiseman, One-sided device-independent quantum key distribution: Security, feasibility, and the connection with steering, Phys. Rev. A 85, 010301 (2012).

[22] T. Gehring, V. Händchen, J. Duhme, F. Furrer, T. Franz, C. Pacher, R. F. Werner, and R. Schnabel, Implementation of continuous-variable quantum key distribution with composable and one-sided-device independent security against coherent attacks, Nat. Commun. 6, 8795 (2015).

[23] N. Walk, S. Hosseini, J. Geng, O. Thearle, J. Y. Haw, S. Armstrong, S. M. Assad, J. Janoušek, T. C. Ralph, T. Symul, H. M. Wiseman, and P. K. Lam, Experimental demonstration of Gaussian protocols for one-sided device-independent quantum key distribution, Optica 3, 634 (2016).

[24] S. Armstrong, M. Wang, R. Y. Teh, Q. H. Gong, Q. Y. He, J. Janousek, H. A. Bachor, M. D. Reid, and P. K. Lam, Multipartite Einstein-Podolsky-Rosen steering and genuine tripartite entanglement with optical networks, Nat. Phys. 11, 167 (2015).

[25] I. Kogias, Y. Xiang, Q. Y. He, and G. Adesso, Unconditional security of entanglement-based continuous-variable quantum secret sharing, Phys. Rev. A 95, 012315 (2017).

[26] Y. Xiang, I. Kogias, G. Adesso, and Q. Y. He, Multipartite Gaussian steering: Monogamy constraints and quantum cryptography applications, Phys. Rev. A 95, 010101(R) (2017).

[27] M. D. Reid, Signifying quantum benchmarks for qubit teleportation and secure quantum communication using EinsteinPodolsky-Rosen steering inequalities, Phys. Rev. A 88, 062338 (2013). 
[28] Q. He, L. Rosales-Zárate, G. Adesso, and M. D. Reid, Secure Continuous Variable Teleportation and Einstein-PodolskyRosen Steering, Phys. Rev. Lett. 115, 180502 (2015).

[29] C.-Y. Chiu, N. Lambert, T.-L. Liao, F. Nori, and C.-M. Li, Nocloning of quantum steering, npj Quantum Inf. 2, 16020 (2016).

[30] M. Piani and J. Watrous, Necessary and Sufficient Quantum Information Characterization of Einstein-Podolsky-Rosen Steering, Phys. Rev. Lett. 114, 060404 (2015).

[31] S.-L. Chen, C. Budroni, Y.-C. Liang, and Y.-N. Chen, Natural Framework for Device-Independent Quantification of Quantum Steerability, Measurement Incompatibility, and Self-Testing, Phys. Rev. Lett. 116, 240401 (2016).

[32] Q. Y. He and M. D. Reid, Genuine Multipartite EinsteinPodolsky-Rosen Steering, Phys. Rev. Lett. 111, 250403 (2013).

[33] T. S. Cubitt, F. Verstraete, W. Dür, and J. I. Cirac, Separable States can be used to Distribute Entanglement, Phys. Rev. Lett. 91, 037902 (2003).

[34] L. Mišta, Jr. and N. Korolkova, Distribution of continuousvariable entanglement by separable Gaussian states, Phys. Rev. A 77, 050302 (2008).

[35] L. Mišta, Jr. and N. Korolkova, Improving continuous-variable entanglement distribution by separable states, Phys. Rev. A 80, 032310 (2009).

[36] A. Fedrizzi, M. Zuppardo, G. G. Gillett, M. A. Broome, M. P. Almeida, M. Paternostro, A. G. White, and T. Paterek, Experimental Distribution of Entanglement with Separable Carriers, Phys. Rev. Lett. 111, 230504 (2013).

[37] C. E. Vollmer, D. Schulze, T. Eberle, V. Händchen, J. Fiurášek, and R. Schnabel, Experimental Entanglement Distribution by Separable States, Phys. Rev. Lett. 111, 230505 (2013).

[38] C. Peuntinger, V. Chille, L. Mišta, Jr., N. Korolkova, M. Förtsch, J. Korger, C. Marquardt, and G. Leuchs, Distributing Entanglement with Separable States, Phys. Rev. Lett. 111, 230506 (2013).

[39] M. Hillery, V. Bužek, and A. Berthiaume, Quantum secret sharing, Phys. Rev. A 59, 1829 (1999).

[40] H.-K. Lau and C. Weedbrook, Quantum secret sharing with continuous-variable cluster states, Phys. Rev. A 88, 042313 (2013).

[41] L. Lami, C. Hirche, G. Adesso, and A. Winter, Schur Complement Inequalities for Covariance Matrices and Monogamy of Quantum Correlations, Phys. Rev. Lett. 117, 220502 (2016).
[42] Note that throughout this Rapid Communication, the (non)steerability is always intended under the experimentally motivated restriction of Gaussian measurements; for steerability of Gaussian states by non-Gaussian measurements, see, e.g., Ref. [43].

[43] Y. Xiang, B. Xu, L. Mišta, Jr., T. Tufarelli, Q. Y. He, and G. Adesso, Investigating Einstein-Podolsky-Rosen steering of continuous-variable bipartite states by non-Gaussian pseudospin measurements, Phys. Rev. A 96, 042326 (2017).

[44] G. Giedke, B. Kraus, M. Lewenstein, and J. I. Cirac, Separability properties of three-mode Gaussian states, Phys. Rev. A 64, 052303 (2001).

[45] See Supplemental Material at http://link.aps.org/supplemental/ 10.1103/PhysRevA.99.010104 for details of the CM of the states generated by the displacement-free optical networks shown in Figs. 2(b) and 4(b), the CM of four-mode states generated in step 4 via a separable ancilla mode, and the way to calculate the optimal displacements exposed on David's mode $D$ to distribute the highest Gaussian steerability between different modes.

[46] R. Simon, Peres-Horodecki Separability Criterion for Continuous Variable Systems, Phys. Rev. Lett. 84, 2726 (2000).

[47] M. D. Reid, Monogamy inequalities for the Einstein-PodolskyRosen paradox and quantum steering, Phys. Rev. A 88, 062108 (2013).

[48] Y. Zhou, X. Jia, F. Li, C. Xie, and K. Peng, Experimental generation of $8.4 \mathrm{~dB}$ entangled state with an optical cavity involving a wedged type-II nonlinear crystal, Opt. Express 23, 4952 (2015).

[49] T. Eberle, V. Händchen, and R. Schnabel, Stable control of $10 \mathrm{~dB}$ two-mode squeezed vacuum states of light, Opt. Express 21, 11546 (2013).

[50] C. Croal, C. Peuntinger, V. Chille, C. Marquardt, G. Leuchs, N. Korolkova, and L. Mišta, Jr., Entangling the Whole by Beam Splitting a Part, Phys. Rev. Lett. 115, 190501 (2015).

[51] A. Rutkowski, A. Buraczewski, P. Horodecki, and M. Stobińska, Quantum Steering Inequality with Tolerance for Measurement-Setting Errors: Experimentally Feasible Signature of Unbounded Violation, Phys. Rev. Lett. 118, 020402 (2017). 